\title{
Gyrotron, a Novel High-Power Microwave Source
}

\author{
M.Q. Tran, Lausanne
}

(Centre de Recherches en Physique des Plasmas, Association Euratom, EPFL)

Recent new applications have stimulated the development of extremely high power sources of electromagnetic (EM) radiation in the centimeter $(\lambda \cong 3 \mathrm{~cm})$ and millimeter $(\lambda \cong 1-3 \mathrm{~mm})$ range. The absorption of electromagnetic waves at the electron cyclotron frequency $\Omega_{\mathrm{e}}=$ $e B_{0} / m_{e^{\prime}}$ or its harmonic $n \Omega_{e^{\prime}}$ is an efficient heating mechanism for electrons in fusion experiments. Moreover, as the resonant absorption layer in the plasma can be defined, the current profile and the plasma pressure gradient can be controlled, which allows us to stabilize unstable magneto-hydrodynamic modes. For present-day or projected machines, the magnetic fields range from $1 T$ to 10T. Hence the corresponding frequencies $\Omega_{\mathrm{e}}$ or $n \Omega_{\mathrm{e}}$ lie between 28 and 300 $\mathrm{GHz}$. Typical powers required are in the range of 1-20 MW for periods varying from $0.1 \mathrm{~s}$ to continuous wave (CW) ${ }^{1}$ ). In a lower frequency band (8-10 GHz), new applications have led to challenging power requirements (1 MW - $600 \mathrm{MW}$ per tube). In the frequency range between 3 and $8 \mathrm{GHz}$, the so-called lower hybrid frequency, plasma heating can again be induced and also a net electrical current generated by transferring the EM wave momentum to the electron. For this, oscillators or amplifiers delivering 0.5-1 $\mathrm{MW}$ in long pulses (up to $10 \mathrm{~s}$ or (W) are required. In the field of high energy physics, high gradient accelerating structures will have to be developed for future $\mathrm{TeV}$ accelerators to re-

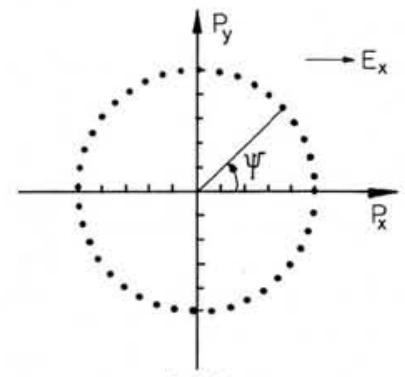

(a) duce their size. Extrapolating from the present day figure of $2.586 \mathrm{GHz}$, it appears that a frequency of $10 \mathrm{GHz}$ would be suitable ${ }^{2}$ ). For this application, amplifiers delivering $300-600 \mathrm{MW}$ in pulses of 100-200 ns would be needed.

In the three applications mentioned, the power and pulse length required are beyond the limits of conventional electron tubes such as klystrons or magnetrons because of ohmic losses on the resonator wall. These are due to an increase in the resistivity $\rho$ at high frequency $f\left(\rho \sim f^{1 / 2}\right)$ and the high electric field $E$. A limit is thus reached and it was to overcome this power limitation that gyrotrons have been developed, first in Gorki in the USSR ${ }^{3}$ ), and then by electron tube companies and research laboratories.

In a gyrotron, the source of free energy is the rotational energy of relativistic electrons in an axial magnetic field $B_{0}$. Electrons guided by $B_{0}$ absorb and emit EM waves at frequencies close to the relativistic electron cyclotron frequency $\Omega_{\mathrm{e}} / \gamma_{0}$ or its harmonic

$$
\omega=n e B_{0} / \gamma_{0} m_{\mathrm{e}}: n=1,2, \ldots
$$

$\gamma_{0}$ is the relativistic factor:

$\gamma_{0}=\left[1+\left(p^{2}+p_{\|}^{2} / m^{2} c^{2}\right]^{1 / 2}\right.$

$$
=\left(1-\left(v^{2}+v_{\|}^{2}\right) / c^{2}\right)^{-1 / 2} \text {. }
$$

The perpendicular and parallel subscripts refer to the directions perpendicular or parallel to $B_{0}$. For simplicity we have assumed that the wave vector $k$ is perpendicular to $B_{0}$, thus avoiding the

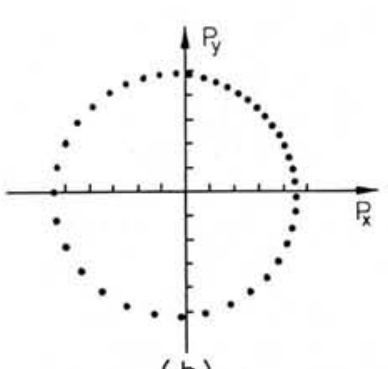

(b)
Fig. $1-$ a) Plot of the electron distribution in the $\left(p_{x}, p_{y}\right)$ plane at the beginning of their interaction with the EM wave. The electrons are uniformly distributed on a circle of radius $p_{\perp}$ $=\left(p_{x}^{2}+p_{y}^{2}\right)^{1 / 2}$. The electric field $\mathrm{E}$ is in the $x$-direction.

b) Due to the relativistic mass variation, electrons are bunched in the positive $p_{x}$ half plane where they lose energy ( $p_{\perp}$ decreases).
Doppler shift in the resonance condition of equ. (1).

Emission and absorption always occur simultaneously, so in order to have a net output, the number of electrons which radiate EM waves must exceed the ones which absorb. This situation is achieved through "azimuthal bunching" and will be outlined for the emission around $\Omega_{\mathrm{e}} / \gamma_{0}$. Only the perpendicular motion is involved. For simplicity we assume that initial electrons have the same perpendicular momentum $p$ and that they are uniformly distributed on a circle of radius $p$ in $\left(p_{x^{\prime}} p_{y}\right)$ space (Fig. 1a). The oscillating electric field $E$ is in the $x$-direction.

Electrons with positive $p_{x}$ lose energy and will thus see their relativistic cyclotron frequency $\Omega_{\mathrm{e}} / \gamma$ increased, while electrons with negative $p_{x}$ will experience the converse. If the wave frequency $\omega$ is larger than $\Omega_{\mathrm{e}} / \gamma_{0}$, there is a bunching of the electrons in the region of the $p_{x}$ plane where they lose their energy (Fig. 1b). Through this process of bunching in the phase angle $\Psi$, we can thus obtain a net energy output. An equivalent way of describing this effect is to state that an electron beam having perpendicular energy is unstable to electromagnetic waves: the instability is known as the "electron cyclotron maser instability". To understand the use of the term "maser", one can refer to the relativistic quantum description of electrons in a magnetic field as described by $\mathrm{J}$. Schneider ${ }^{4}$ ). The energy level of an electron in a magnetic field is

$E_{i}=m_{0} c^{2}\left[1+2(i+1 / 2) \hbar \Omega_{\mathrm{e}} / m_{0} c^{2}\right]^{1 / 2}$ $-m_{0} c^{2}$.

In the presence of an RF field the electron population will undergo absorption and induced emission. A net power transfer from the electrons to the wave is shown to occur if $\omega>\omega_{i, i+1}$, where $\omega_{i, i+1}$ is the frequency of the photon emitted during the transition $i+1$ to $i$. As pointed out by Schneider ${ }^{4}$ ), "it does not appear unlikely that this effect could be used for a new type of maser". In the remaining part of this presentation, we shall try to outline how this prediction came to be realised. 


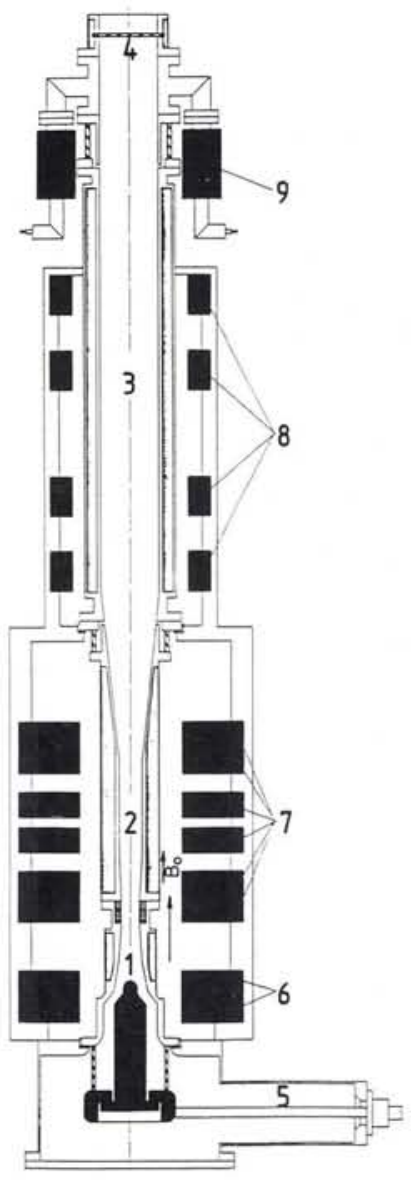

Fig. 2 - Schematic of a gyrotron with cylindrical cavity (Courtesy of Brown, Boveri and Cie., Dept. EKR).

1. electron gun

2. resonator

3. collector

4. $R F$ window

5. $\mathrm{SF}_{6}$ insulation hood

6. gun magnet coils

7. resonator magnet coils

8. collector coils

9. ion getter pumps.

As in all oscillators, the energy transfer between the EM wave and the source of free energy (here the electron beam guided by the static magnetic field $B_{0}$ ) requires a high quality factor structure, which could be either a microwave cavity or a quasi-optical (Q.O.) Fabry-Perot resonator. Since the frequency of the electron cyclotron maser instability is determined by the magnetic field $B_{0}$ ( $c f$ Eq. 1), the use of an overmoded cavity or resonator can be considered. This freedom is used by designers to optimize the efficiency, $\eta_{\mathrm{el}}$, of the energy transfer from the electron beam to the EM wave, while keeping the ohmic losses in the wall of the resonant structure within an acceptable limit $\left(<1.5 \mathrm{~kW} / \mathrm{cm}^{2}\right)$ using standard cooling methods already tested in high power tubes. $\eta_{\text {el }}$ is defined by $\eta_{\mathrm{el}}=\left[\langle\gamma\rangle_{\mathrm{z}=0}-\langle\gamma\rangle_{\mathrm{z}=\mathrm{L}}\right] /$

$$
\left[\langle\gamma\rangle_{z=0}-1\right]
$$

where the average $<\cdot>$ is made over all the phase angle of the electron and $L$ is the length of the zone where the electrons interact with the EM field. The $\gamma$ of each particle is obtained by integration of the relativistic Newton law

$$
\begin{aligned}
d p / d t= & -e\left[E(z, \omega)+\boldsymbol{v} \times \boldsymbol{B}_{0}+\right. \\
& \boldsymbol{v} \times \boldsymbol{B}(z, \omega)]
\end{aligned}
$$

$E(z, \omega)$ and $B(z, \omega)$ are the wave electric and magnetic fields and depend on the geometry of the specific resonant structure.

A schematic of a gyrotron with a conventional microwave cavity operating in the $\mathrm{TE}_{m n 1}$ mode is shown in Fig. 2. A suitable electron beam (i.e. one having a large perpendicular energy component, and a low dispersion in both energy $\gamma$ and pitch angle) is guided by the static magnetic field $B_{0}$ into the cavity. Its dimensions are designed so that the resonant frequency of the desired mode corresponds to the one given by equation (1). The parallel wave vector $k_{\|}$is close to zero and the Doppler shift effect on the resonance condition is cancelled, minimizing the deleterious effect of pitch angle spread. The mode indexes $(m, n)$ are selected to keep the thermal loading on the wall within the abovementioned limits: in the frequency range of $100 \mathrm{GHz}$ and at a power level of a few hundred kilowatts, symmetric modes $\mathrm{TE}_{\mathrm{O} n}(n<4)$ are generally considered. At higher frequency ( $\geq 100 \mathrm{GHz}$ ) and/or higher power $(\gtrsim 200 \mathrm{~kW})$ asymmetric modes are necessary in order to avoid excessive ohmic losses in the cavity. Larger size cavities are also required at high power to permit the use of an electron beam with larger cross-sections. This last requirement is necessary to avoid a too important space charge in the beam at high current $(I \cong 50-100 \mathrm{~A}$, $j \cong 600 \mathrm{~A} / \mathrm{cm}^{2}$ ). With strongly overmoded cavities, a careful design of the cavity geometry is necessary to avoid the problem of competition between various modes, the resonant frequencies of which could be close enough to be within the unstable frequency domain of the electron cyclotron maser instability. Gyrotrons using cylindrical cavities are now commercially available in the frequency range from $28 \mathrm{GHz}$ up to 140 $\mathrm{GHz}$, delivering powers of $100-200 \mathrm{~kW}$ continuous wave. At the Massachusetts Institute of Technology, a gyrotron has been tested with powers up to $650 \mathrm{~kW}$ between 126 and $245 \mathrm{GHz}$ in short pulse $\left.{ }^{5}\right)$. In the lower end of the frequency range $(8-10 \mathrm{GHz})$ development programmes are aiming towards 500-1000 $\mathrm{kW} \mathrm{CW}{ }^{6}$ ) and $30 \mathrm{MW}$ in $\mu$ s pulses ${ }^{2}$ ).

A second class of gyrotrons is now being considered, the Q.O. gyrotrons (Fig. 3). In Fig. 3 one recognizes the main

\section{Second International Conference on Tethers in Space}

October 4-8, 1987

Venice, Italy

Sponsored by: PSN

Piano Spaziale Nazionale - CNR

NASA

National Aeronautics and Space Administration

ESA

European Space Agency

The objective of the Conference is to provide a focus on how tether systems may be used for science in the era of the Space Station.

For further information please contact:

Dr. Alberto Loria

Piano Spaziale Nazionale - CNR

Viale Regina Margherita 202

I - 00198 Roma

Tel. (6) 4767250

Telex 616162 CNRPSN I

Telefax (6) 4767267

components of a gyrotron (the electron gun and the magnetic field coils) but the cylindrical microwave cavity with its axis parallel to $B_{0}$ is replaced by a FabryPérot resonator with its axis perpendicular to the magnetic field lines. This

\section{EPF - LAUSANNE}

Applications are invited for a post doctoral position at the Institute of Micro- and Optoelectronics at the Ecole Polytechnique Fédérale (EPF) in Lausanne - Switzerland.

The research connected with this position involves experimental investigations of $\mathrm{GaAs} / \mathrm{AIGaAs}$ quantum Hall devices for metrological applications, including materials growth and sample preparation aspects as well as high precision measurement techniques. The work will be carried out in collaboration with the Swiss Federal Office of Metrology in Bern where the measurement apparatus is located.

Candidates should have a recent $\mathrm{PhD}$ and research experience in the above mentioned field. The duration of the appointment is two years, with an extension possible. The salary will be approx. SFR 60000.- to 70000.- depending on experience.

Letters of application, including a curriculum vitae with list of publications and a brief description of research interest should be addressed to:

Prof. M. llegems,

Institute of Micro- and Optoelectronics, EPFL, Dept. of Physics,

$\mathrm{CH}$ - 1015 Lausanne. 
ensures a zero parallel wave vector in the interaction region and reduces the dependency of the output efficiency of the gyrotron on the pitch angle variation. Moreover this geometry leads to a natural separation between the EM output and the spent electron beam: high power collectors for the spent electron beam can be designed without any constraints from the microwave-guided propagation.

The resonator is formed by two mirrors, separated by a distance $d$. The mirrors are characterized by two dimensionless parameters, $g_{i}$ and the Fresnel number $N_{i}$

$$
\begin{aligned}
& g_{i}=1-d / R_{i} \quad \mathrm{i}=1,2 \\
& N_{i}=a_{i}^{2} / \lambda d
\end{aligned}
$$

where $R_{i}, a_{i}$ and $\lambda$ are respectively the radius of curvature, the mirror radius and the wavelength at which the resonator operates.

The resonator parameters are optimized to yield the highest efficiency $\eta_{\text {tot }}$ of energy transfer between the electron beam and the EM output radiation under the main constraint of a maximum ohmic loss on the mirror ( $\cong 1.5 \mathrm{~kW}$ / $\mathrm{cm}^{2}$ ). The total efficiency $\eta_{\text {tot }}$ in this case must include not only the electronic efficiency $\eta_{\text {el }}$ defined previously but also $\eta_{\text {out, }}$ the efficiency of coupling out the EM from the resonator to the output guide. The total efficiency is then given by:

$$
\eta_{\text {tot }}=\eta_{\mathrm{el}} \cdot \eta_{\text {out }}
$$

Fig. 3-Schematic of a quasi-optical gyrotron [Ref. 8].

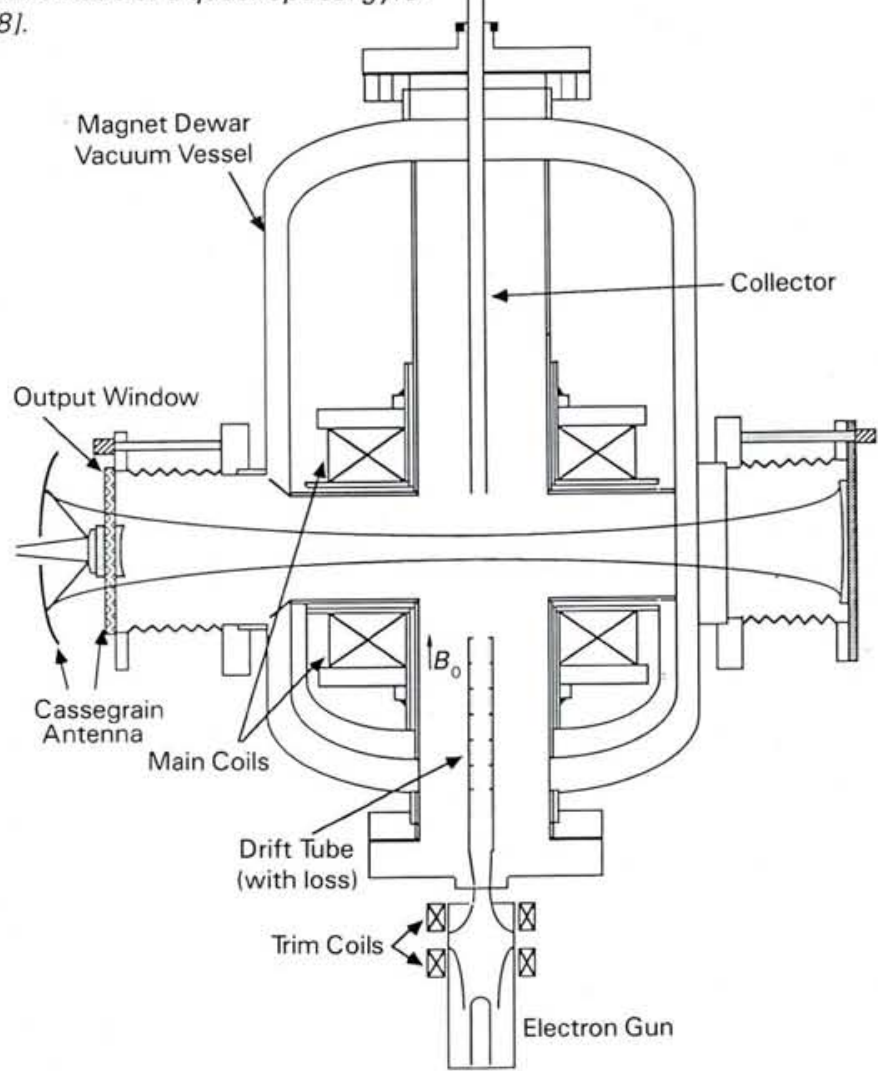

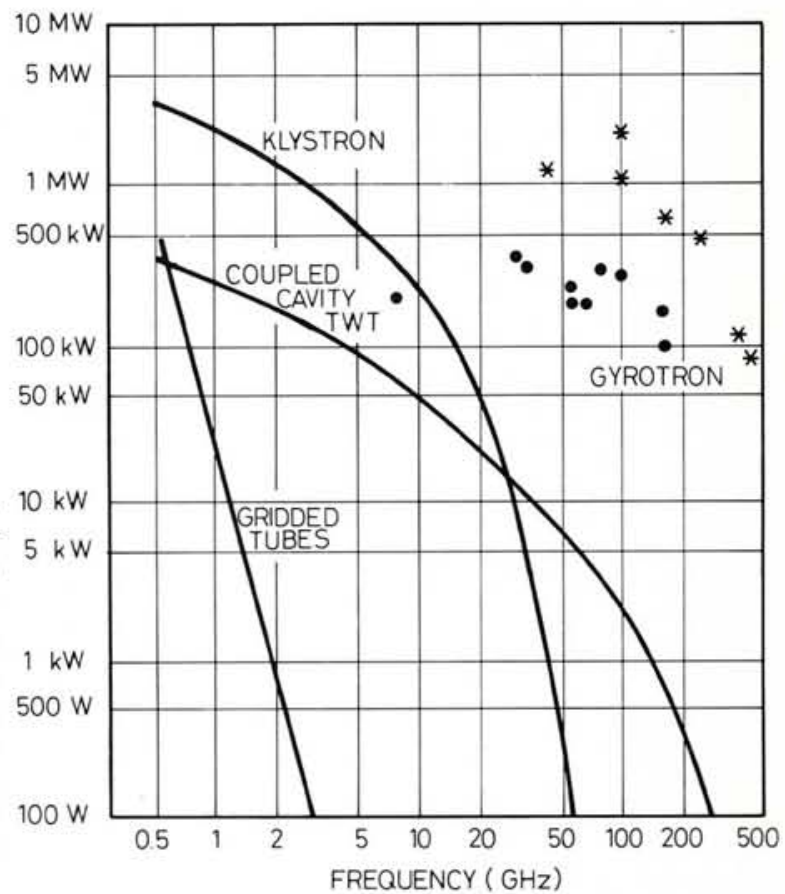

$\eta_{\text {out }}$ is defined as the ratio between the power collected through the output coupling structure and the total power lost by the resonator. Its value which is less than unity (only a fraction of the total power diffracted by the mirrors can be guided through it) is readily computed using the Fresnel Huygens theory $\left.{ }^{8}\right)$ of resonators. In presently designed experiments, $\eta_{\text {out }}$ is around $80-95 \%$. The electronic efficiency is computed

using (2) and Newton's equation (3) With the constraint of a maximum permissible heat load around $1.5 \mathrm{~kW} / \mathrm{cm}^{2}$ on the mirror, $\eta_{\mathrm{el}}$ is typically around $25-30 \%$ with an annular beam of $70 \mathrm{kV}$ and $p / p_{\|}=1.5$. Typically a mirror separation $d$ of 50 to $75 \mathrm{~cm}$ is used, the large $d$ being necessary to increase the EM spot size on the mirror and thus reduce the peak heat load. Although calculations indicate that large negative $g$ values $(-0.9<g<-0.5)$ improve $\eta_{\mathrm{el}}$ one should avoid being too close to the stability limit of the resonator and we find $|g|>1$.

The phase angle of the electrons from the beam is uniformly distributed over $2 \pi$ when they enter the resonator, but the electronic efficiency can be increased if the electrons reach the interaction region correctly bunched in a slowly varying phase angle. This can be achieved using a "prebunching" resonator separated from the energy extracting resonator by a drift space. The scheme is then analogous to the familiar two cavities klystron, hence the name of "QuasiOptical Gyroklystron". In the prebuncher, the oscillating electric field produces a small change in perpendicular momentum, which causes a slow shift in the phase angle in the drift space and the electrons enter the energy extracting resonator already correctly bunched in $\left(p_{x^{\prime}}, p_{y}\right)$ space.

Since the resonator is strongly overmoded, the separation between two consecutive $\mathrm{TEM}_{\mathrm{OOq}}$ modes (equal to $c / 2 d \cong 200 \mathrm{MHz}$ with $d \cong 75 \mathrm{~cm}$ ) is much smaller than the bandwidth of the cyclotron maser instability $(\sim 3 \mathrm{GHz})$. Because of this, the Q.O. gyrotron has a 
greater tendency to oscillate at more than one frequency. As a result $\eta_{\mathrm{el}}$ could be lower than for a single frequency and the window must be matched over a large bandwidth which complicates its design especially at high power. The growth of all the unstable EM waves and their subsequent non-linear evolution has been studied numerically by $A$. Bondeson et al. ${ }^{7}$ ). Starting from the noise level, typically a self-consistent time evolution of around 20 modes, separated in frequency by $c / 2 d$, is followed. The calculation is self-consistent in the sense that the oscillating current $j(\omega)$ which creates the EM field results from the interaction of the electron beam with the EM field itself. Single mode operation could be achieved however, with a suitable profile of $B_{0}$ and mode selectivity should be improved.

The potentialities of a quasi-optical gyrotron are not yet fully assessed. Results from the Naval Research Laboratories presented at a recent meeting ${ }^{8}$ ) have provided confirmation of the numerical predictions of the code of Bondeson et al. in regard to both the frequency spectrum and its temporal evolution. However, the measured total efficiency was low $\left(\eta_{\text {tot }} \cong 6 \%\right.$ ) and this has been attributed to the low perpendicular energy of the beam and the large loss in the coupling efficiency ( $\eta_{\text {out }} \cong 53 \%$ ). Further experiments are now in preparation in the US as well as in our own laboratory.

The performance of present gyrotrons are summarized and compared to other electron tubes in Fig. 4. We have restricted ourselves in this survey to devices which use weakly relativistic electron beams (beam energy $E_{\mathrm{b}}<100 \mathrm{keV}$ ) although gyrotrons with $E_{\mathrm{b}}>200 \mathrm{keV}$ have been tested and give an output power in the range of $10 \mathrm{MW}-1 \mathrm{GW}$ in the frequency range of $8 \mathrm{GHz}$ to 120 $\mathrm{GHz}$. In Fig. 4 we have distinguished between short pulse devices (pulse length $<5 \mathrm{~ms}$ ) and longer pulse tubes (pulse length $>5 \mathrm{~ms}$ ) based on the published results. It is worth noting that many of the short pulse gyrotrons have $\mathrm{CW}$ features but due to the limitation in the experimental facilities, the tests have only been run on short pulses. Fig. 4 clearly shows that gyrotrons can exceed the power limitation of conventional tubes at high frequency ( $>10 \mathrm{GHz}$ ).

While describing the important issues related to the two concepts presently used in the design of gyrotrons, we have limited ourselves to oscillators only. It is worth noting that amplifiers based on the electron cyclotron maser instability have also been designed: multi-cavities gyroklystrons, gyrotravelling wave amplifiers are the counterparts of the well-known existing electron tubes.

\section{REFERENCES}

1. Riviere A.C., Int. J. Electronics 61 (1986) 693.

2. Granatstein V.L., Vitello P., Chu K.R., Ko K., Latham P.E., Lawson W., Striffler C.D. and Drobot A., IEEE Trans. Nuclear Sci. NS32 (1985) 2957.

3. See for example Flyagin V.A., Gapanov A.V., Petelin M.I. and Yulpatov V.K., IEEE Trans. Microwave Theory Tech. MIT25 (1977) 514.

4. Schneider J., Phys. Rev. Lett. 2 (1959) 504.

5. Kreischer K. and Temkin R.J., Plasma Fusion Center, MIT, Report PFC/JA-87-15 (April 1987).

6. Mathews H.G., Alberti S., Muggli P., Perrenoud A. and Tran M.Q., IEDM Tech. Digest 86 (1986) 805.

7. Bondeson A., Manheimer W. and Ott E., Infrared and Millimeter Waves, Ed. K.J. Button (Academic Press) 9309.

8. Read M.E., Tran M.Q., Mc Adoo J., Hargreaves T., Barsanti M., Lee R. and Manheimer W.M., 11th Int. Conference on Infrared and Millimeter Waves, 1986, Pisa.

\section{Joint Prizes in Physics 1987}

The European Physical Society is exceptional in awarding only one prize for physics per year (the Hewlett-Packard Europhysics Prize); the majority of our member organizations have a regular series. Often these commemorate the work of a famous physicist, and have been created to perpetuate the name of an illustrious past member. The total number awarded each year in Europe is difficult to estimate, as some receive wide publicity, at least in the specialist domain concerned, others are known only locally. The figure is certainly many tens.

It would clearly be impossible for Europhysics News to give even a brief review of the reasons behind all these and the careers of those who have been honoured, yet prizes do represent a real comment on recent key research as well as defining who and where this research was done. This is particularly true when the prize awarded is international. The Nobel Prize has for many years been regarded as the ultimate accolade, but there are other prestigious international prizes in physics, notably in Europe the joint paired prizes of the Deutsche Physi- kalische Gesellschaft, the Société Francaise de Physique, and The Institute of Physics of the UK.

These are as follows, listed in the order in which they made their appearance: the HOLWECK prize which was instituted in 1945 by the French and British physical societies; the MAX BORN prize instituted in 1972 by the British and German physical societies, and finally the GENTNER-KASTLER prize instituted by the German and French physical societies, in 1985. The first awards were respectively presented in 1946, 1973 and 1986. The list of recipients today counts 43 names for the Holweck prize, 15 names for the Max Born prize and two names for the Gentner-Kastler prize.

The procedures used for the three awards are almost the same. The Council of one society selects the winner from a list of typically three names presented by the other society, the respective roles of the two societies alternating from year to year. In all three cases, the award consists of a medal and a certificate which is accompanied by a cash prize. The value is $€ 300$ for the Holweck and Max Born prizes and 1000 ECU for the Gentner-Kastler prize, reference to the European Accounting Unit being a sign of its relatively later institution. The key point, however, is that these prizes carry a very high prestige to which the list of past recipients bears clear witness. All three societies consider these joint prizes as among the very few top prizes which they award each year.

Direct links between different physical societies are very important to the harmonious development of physics in Europe. The co-ordination provided by the EPS is strengthened by the numerous and efficient bilateral or multilateral links. Such contacts can take various forms but awarding joint prizes is an efficient way to generate them. This implies regular contacts between officials of two societies which can but naturally develop into other joint studies, activities and ventures. One can only hope that these joint prizes, which are at present limited to the France-Federal Republic of Germany and United Kingdom triangle, will increase in number. However, in order to avoid a prize inflation, this may involve the redefinition of some 